\title{
On the Impact of the Relativistic Acceleration of ENET on the Electromagnetic Analogy of Gravitational Orbits' Decay
}

\author{
Barbaro Quintero-Leyva \\ Independent Work, Miami, FL, USA \\ Email: doserate2002@yahoo.com
}

How to cite this paper: Quintero-Leyva, B. (2019) On the Impact of the Relativistic Acceleration of ENET on the Electromagnetic Analogy of Gravitational Orbits' Decay. Open Access Library Journal, 6: e5673.

https://doi.org/10.4236/oalib.1105673

Received: August 7, 2019

Accepted: August 23, 2019

Published: August 26, 2019

Copyright (C) 2019 by author(s) and Open Access Library Inc.

This work is licensed under the Creative Commons Attribution International License (CC BY 4.0).

http://creativecommons.org/licenses/by/4.0/

\begin{abstract}
The Kepler $3^{\text {rd }}$ law (K3L) was extended considering the gravitational field, in analogy to electricity and magnetism (E\&M), produced from the circular motion of point masses of a binary system. Additionally the Newtonian acceleration in the field equation was replaced with the relativistic acceleration (ENET). It was found out that the increase of the angular speed with the decrease of the mass separation is less drastic for the E\&M analogy than for the K3L case. The use of the ENET acceleration however yielded a more drastic profile than the K3L results. A gravitational model (in analogy to $\mathrm{E} \& \mathrm{M}$ ) for circular orbits reported previously was extended to cover relativistic speeds. It was found out that even for low speeds the linear-momentum term was not negligible. The extended model yielded faster orbital decay than the non-extended one. The use of the extended K3L using ENET yielded even faster orbit decay but slower than the results of the linearized general theory of relativity (LGR). Similar results were obtained when the gravitational radiation fields were compared. The period decay (for circular orbit) of the Hulse and Taylor pulsar binary was simulated; the extended models yielded larger period decay than the non-extended one but smaller than the results of LGR.
\end{abstract}

\section{Subject Areas}

Classical Physics, Special Theory of Relativity

\section{Keywords}

Newtonian Gravitation, Newton's $2^{\text {nd }}$ Law, Theory of Relativity, Electromagnetism, Gravitational Waves 


\section{Introduction}

The similarity between the Coulomb's law for electrostatics and Newton's law for gravitation triggers the question: Could the laws of electrodynamics be extrapolated to gravitation? Efforts in this direction have been made before by, for example, Maxwell and Heaviside in the 1800's. One characteristic of classical electrodynamics is the generation of electromagnetic waves by accelerated charges which when it was experimentally demonstrated it revolutionized the communications and it is still the corner stone of the modern communication devices. There have been many attempts to develop gravitational models that generate gravitational waves in addition to the ingenious general theory of relativity (GR) of Einstein. The reasons for that, apart from just curiosity, could be: The complexity of the math (and also the physical concepts) involved in GR which can be avoided (and still giving students sound basis for understanding the wave generation process) using for example a gravitational analogy to classical electrodynamics, see for example [1] [2], where a gravitational model, in analogy to E\&M, was developed for a binary system composed of point masses which produces relations in striking agreement with the LGR dependencies. The amplitudes of the relations are however significantly lower than the LGR's ones. Another reason for developing other theories is the belief that GR is not the ultimate (if such a theory could ever be developed) theory, especially, if the problems of dark matter and dark energy (or alternatively dark physics) are considered. Note that [3] found that GR could be ruled out, in favor of a vector theory of gravity, based on the ratio of the strain signal of the three interferometers of LIGO-VIRGO from the GW170817 event.

In this work an attempt was made to implement the suggested correction mentioned in [1] to the gravitational potentials to consider its impact on the decay of a circular orbit. Extensions of the $3^{\text {rd }}$ Kepler's law (K3L) were derived and their impact on the orbit decay was also assessed. The objective of this work was to determine the impact of ENET [4] on the electromagnetic analogy of gravitational orbits' decay.

\section{Gravitational Decay of Circular Orbits as an Analogy of Classical Electromagnetism}

\subsection{Extension of Kepler's $3^{\text {rd }}$ Law Using Gravitational Fields Analog to the Classical Electromagnetic Fields}

In analogy to the Lorentz force of classical electromagnetism, the gravitational force acting on a point mass $m_{a}$ due to another point mass $m_{b}$ can be formulated which when equated to the inertial force ( $2^{\text {nd }}$ law of Newton), the following balance in polar coordinates is obtained:

$$
\boldsymbol{F}_{b a}=-m_{a}\left(\boldsymbol{E}_{b a}+\boldsymbol{v}_{a} \times \boldsymbol{B}_{b a}\right)=m_{a} \boldsymbol{a}_{a}=m_{a}\left(\left(\ddot{r}_{a}-r_{a} \dot{\theta}_{a}^{2}\right) e_{r_{-} a}+\left(r_{a} \ddot{\theta}_{a}+2 \dot{r}_{a} \dot{\theta}_{a}\right) e_{\theta_{-} a}\right)
$$

where

$$
\boldsymbol{E}_{b a}: \text { Gravitational equivalent of the electric field acting on body a }
$$


$\boldsymbol{v}_{a}:$ Velocity of body a

$\boldsymbol{B}_{b a}$ : Gravitational equivalent of the magnetic field acting on body a

$\boldsymbol{a}_{a}$ : Acceleration of body a

$r_{a}, \theta_{a}:$ Radial and azimuthal coordinates of body a respectively

$e_{r_{-} a}, e_{\theta_{-} a}:$ Radial (pointing from the origin of the laboratory reference frame to a) and azimuthal (counter clockwise from $x$ to $y$ ) unit vectors of body a respectively

The number of dots on top of a variable represents the order of the time derivative.

The polar unit vectors are time and body dependent, unlike Cartesian unit vectors $(\hat{\dot{x}}, \hat{y})$, which can be written as

$$
e_{r_{-} a}=\hat{\dot{x}} \cos \theta_{a}(t)+\hat{y} \sin \theta_{a}(t), e_{\theta_{-} a}=-\hat{\dot{x}} \sin \theta_{a}(t)+\hat{y} \cos \theta_{a}(t)
$$

Note the minus sign in Equation (1) which is absent in electromagnetism (where the character of the force is determined by the sign of charges). This modification is made based on the still holding experimental evidence that the gravitational force is attractive in nature. By making this modification here, the need for defining a negative gravitational constant $(G)$ is avoided.

$\boldsymbol{E}_{b a}$ is determined using $G$ in place of $\frac{1}{4 \pi \varepsilon_{0}}$ in equation 10.65 of [5]:

$$
\begin{gathered}
\boldsymbol{E}_{b a}=G \frac{m_{b} r_{b a}}{\left(\boldsymbol{r}_{b a} \cdot \boldsymbol{u}_{b a}\right)^{3}}\left[\left(c^{2}-v_{b}^{2}\right) \boldsymbol{u}_{b a}+\boldsymbol{r}_{b a} \times\left(\boldsymbol{u}_{b a} \times \boldsymbol{a}_{b}\right)\right] \\
\boldsymbol{B}_{b a}=\frac{1}{c} \hat{r}_{b a} \times \boldsymbol{E}_{b a}
\end{gathered}
$$

where,

$$
\begin{gathered}
r_{b a}=\left|\boldsymbol{r}_{a}(t)-\boldsymbol{r}_{b}\left(t_{b a}\right)\right|=c\left(t-t_{b a}\right), \quad \boldsymbol{r}_{b a}=\boldsymbol{r}_{a}(t)-\boldsymbol{r}_{b}\left(t_{b a}\right), \quad \boldsymbol{u}_{b a}=c \hat{r}_{b a}-\boldsymbol{v}_{b}\left(t_{b a}\right) \\
\hat{r}_{b a}=\boldsymbol{r}_{b a} / r_{b a}, \quad \boldsymbol{v}_{b}=\mathrm{d} \boldsymbol{r}_{b}\left(t_{b a}\right) / \mathrm{d} t, \quad v_{b}=\left|\boldsymbol{v}_{b}\right|, \quad \boldsymbol{a}_{b}=\mathrm{d} \boldsymbol{v}_{b}\left(t_{b a}\right) / \mathrm{d} t
\end{gathered}
$$

c. Speed of light in vacuum

$r_{b a}$ : Separation distance between point mass $a$ and $b$

$t_{b a}$ : Retarded time: Time needed to transmit the action (information) of the force from body $b$ to body $a$

Assuming that the point masses follow circular orbits with piece-wise constant angular speed $(\omega)$ the following is obtained for the fields acting on mass $a$ :

$$
\begin{gathered}
\boldsymbol{E}_{b a}=G \frac{m_{b}}{(r c)^{2}}\left[\left(\left(\omega \cdot r_{b}\right)^{2}-c^{2}\right) e_{r_{b}}\left(t_{b a}\right)+\left(f / c-c \omega \cdot r_{b}\right) e_{\theta_{b}}\left(t_{b a}\right)\right] \\
\boldsymbol{B}_{b a}=G \frac{m_{b}}{(r c)^{2}} \frac{1}{c}\left[\left(-f / c+c \omega \cdot r_{b}\right) e_{z}\right] \\
f=\omega^{3}\left(r_{b}^{3}-r r_{b}^{2}\right)
\end{gathered}
$$

where,

$r=r_{b a}:$ Separation distance between the point masses 
$\omega:$ Angular speed of body $b$

$e_{r_{-} b}\left(t_{b a}\right), e_{\theta_{-} b}\left(t_{b a}\right):$ Radial unit vector (pointing from the origin of the laboratory reference frame to $b$ ) and azimuthal (counter clockwise, from $x$ to $y$ ) unit vector respectively.

$$
e_{r_{-} b}\left(t_{b a}\right)=-\hat{\dot{x}} \cos \left(\omega t_{b a}\right)-\hat{y} \sin \left(\omega t_{b a}\right), e_{\theta_{-} b}\left(t_{b a}\right)=\hat{\dot{x}} \sin \left(\omega t_{b a}\right)-\hat{y} \cos \left(\omega t_{b a}\right)
$$

Note that Equations (4) and (5) constitute the solution of a variant of the problem 10.20 of [5]. See [6] for the solution of the original problem. Note that the sign of the unit vectors were changed for consistency with the gravitational wave section.

Equation (1) for circular orbit with a constant angular speed can be written as:

$$
-\left(\boldsymbol{E}_{b a}+\boldsymbol{v}_{a} \times \boldsymbol{B}_{b a}\right)=-r_{a} \omega^{2} e_{r_{-} a}=-\frac{v_{a}^{2}}{r_{a}} e_{r_{-} a} \text { (7) } \omega=\dot{\theta}
$$

Substituting Equation (4) and (5) into Equation (7), considering small $r$ $\left(e_{r_{-} b}\left(t_{b a}\right) \approx e_{r_{-} b}(t)\right)$, a CM reference frame $\left(e_{r_{-} a}(t)=-e_{r_{-} b}(t)\right)$ and ignoring in the left hand side the dependency on $e_{\theta_{-} b}$ (the right hand side of Equation (7) does not depend on $e_{\theta_{-} b}$ ) leads to:

$$
-G \frac{m_{b}}{(r c)^{2}}\left(\left(\omega \cdot r_{b}\right)^{2}-c^{2}-\frac{\omega \cdot r_{a} f}{c^{2}}+\omega^{2} \cdot r_{a} r_{b}\right)=\frac{\left(\omega \cdot r_{a}\right)^{2}}{r_{a}} \text {, from which the fol- }
$$

lowing is obtained:

$$
-\frac{\eta_{b}\left(\eta_{a}^{3}-\eta_{a}^{2}\right) r^{4}}{c^{4}} \omega^{4}+\left(\frac{\left(\eta_{b} \eta_{a}+\eta_{a}^{2}\right) r^{2}}{c^{2}}+\frac{r^{3}}{G M}\right) \omega^{2}-1=0
$$

where, $\eta_{a}=m_{a} / M, \eta_{b}=m_{b} / M, M=m_{a}+m_{b}$.

The CM relations $\boldsymbol{r}_{a}=\frac{m_{b}}{m_{a}+m_{b}} \boldsymbol{r}$ and $\boldsymbol{r}_{b}=-\frac{m_{a}}{m_{a}+m_{b}} \boldsymbol{r}$ (9), were used to obtain Equation (8).

Note that making $c=\infty$ Kepler's $3^{\text {rd }}$ law for circular orbits is recovered. Equation (8) is solved using the substitution $\omega^{\prime}=\omega^{2}$.

\subsection{Gravitational Wave as an Analogy to the Classical Electromagnetic Waves}

In this section the model presented in [1] is classically (prior Einstein's relativity) extended to speeds comparable to the speed of light in vacuum following the correction therein indicated which contains the ratio of the binary velocity to the speed of light, the resultant equations constitute the gravitational equivalent of the Lienard-Wiechert potentials.

The gravitational fields can also be written as [5] $\boldsymbol{E}=-\nabla \Phi-\frac{\partial \boldsymbol{A}}{\partial t}$ and $\boldsymbol{B}=\nabla \times \boldsymbol{A}$

where, $\Phi, \boldsymbol{A}$ are the scalar and vector potential respectively. For the binary system in question the superposition of the gravitational-equivalent Lienard-Wiechert potentials at the observation point $R$ are written as 


$$
\begin{gathered}
\Phi(\boldsymbol{R}, t)=\frac{G m_{a}}{R_{a}-\boldsymbol{R}_{a} \cdot \boldsymbol{v}_{a} / c}+\frac{G m_{b}}{R_{b}-\boldsymbol{R}_{b} \cdot \boldsymbol{v}_{b} / c} \\
\boldsymbol{A}(\boldsymbol{R}, t)=\frac{G}{c^{2}} \frac{m_{a} \boldsymbol{v}_{a}}{R_{a}-\boldsymbol{R}_{a} \cdot \boldsymbol{v}_{a} / c}+\frac{G}{c^{2}} \frac{m_{b} \boldsymbol{v}_{b}}{R_{b}-\boldsymbol{R}_{b} \cdot \boldsymbol{v}_{b} / c}
\end{gathered}
$$

where, $R_{a}, R_{b}$ are the distances from mass $a$ and $b$ respectively, to the observation point. The positions and velocities are evaluated at their respective retarded time: $t_{R a}=t-R_{a} / c$ and $t_{R b}=t-R_{b} / c$.

Note that the gravitational constant is kept positive in the potentials. The attractive character of the gravitation was considered in the gravitational-equivalent Lorentz force.

The positions and velocities of the components of the binary system moving in circular orbit with piece-wise constant angular speed in the CM reference frame are written as

$$
\begin{gathered}
\boldsymbol{r}_{a}=r_{a}\left[\hat{x} \cos \left(\omega \cdot t_{R a}\right)+\hat{y} \sin \left(\omega \cdot t_{R a}\right)\right] \\
\boldsymbol{r}_{b}=-r_{b}\left[\hat{x} \cos \left(\omega \cdot t_{R b}\right)+\hat{y} \sin \left(\omega \cdot t_{R b}\right)\right] \\
\boldsymbol{v}_{a}=\omega \cdot r_{a}\left[-\hat{x} \sin \left(\omega \cdot t_{R a}\right)+\hat{y} \cos \left(\omega \cdot t_{R a}\right)\right] \\
\boldsymbol{v}_{b}=\omega \cdot r_{b}\left[\hat{x} \sin \left(\omega \cdot t_{R b}\right)-\hat{y} \cos \left(\omega \cdot t_{R b}\right)\right]
\end{gathered}
$$

and

$$
\begin{gathered}
\boldsymbol{r}=r\left[\hat{x} \cos \left(\omega \cdot t_{R}\right)+\hat{y} \sin \left(\omega \cdot t_{R}\right)\right] \\
t_{R}=t-R / c
\end{gathered}
$$

The gravitational radiation field is determined as $\boldsymbol{g}_{\text {rad }}=-\frac{\partial \boldsymbol{A}_{\text {Trans }}}{\partial t}$ which depends only on the components of the vector potential $\boldsymbol{A}_{\text {Trans }}$ that are transverse to the observation direction. The power radiated per unit area in the direction of a monochromatic wave propagation is determined as $\boldsymbol{S}=\frac{c}{4 \pi G} \boldsymbol{g}_{\text {rad }}^{2}(t) \hat{S}$. Note that in this work the average value, which is optional in EM and required in GR [2]:, is not used for $S$.

Expanding in Taylor series the velocities around $t_{R}$ Equation (11) becomes:

$$
\begin{array}{r}
\boldsymbol{A}(\boldsymbol{R}, t)=\frac{G}{c^{2}} \frac{m_{a}\left[\boldsymbol{v}_{a}\left(t_{R}\right)+\left.\frac{\mathrm{d} \boldsymbol{v}_{a}}{\mathrm{~d} t}\right|_{t_{R}} \Delta t_{a}\right]}{R_{a}-R_{a} \hat{R}_{a} \cdot\left[\boldsymbol{v}_{a}\left(t_{R}\right)+\left.\frac{\mathrm{d} \boldsymbol{v}_{a}}{\mathrm{~d} t}\right|_{t_{R}} \Delta t_{a}\right] / c} \\
\left.+\left.\frac{m_{b}\left[\boldsymbol{v}_{b}\left(t_{R}\right)+\left.\frac{\mathrm{d} \boldsymbol{v}_{b}}{\mathrm{~d} t}\right|_{t_{R}} \Delta t_{b}\right]}{c^{2}} \frac{\mathrm{d} \boldsymbol{v}_{b}}{\mathrm{~d} t}\right|_{t_{R}} \Delta t_{b}\right] / c
\end{array}
$$

Using the binomial expansion in the law of cosines relating $R_{a}, R_{b}$ with 
$r_{a}, r_{b}$, considering $R \gg r_{a}, r_{b}$ and assuming that $R \approx R_{a} \approx R_{b}$ (only for the quantities explicitly written in the denominator) the following is obtained:

$$
\begin{gathered}
A(\boldsymbol{R}, t)=\frac{G}{c^{2} R} \frac{m_{a}\left[\boldsymbol{v}_{a}\left(t_{R}\right)+\left.\frac{\mathrm{d} \boldsymbol{v}_{a}}{\mathrm{~d} t}\right|_{t_{R}} \Delta t_{a}\right]}{d e n_{a}}+\frac{G}{c^{2} R} \frac{m_{b}\left[\boldsymbol{v}_{b}\left(t_{R}\right)+\left.\frac{\mathrm{d} \boldsymbol{v}_{b}}{\mathrm{~d} t}\right|_{t_{R}} \Delta t_{b}\right]}{d e n_{b}} \\
\operatorname{den}_{a}=1-\frac{1}{c} \hat{R}_{a} \cdot\left[\boldsymbol{v}_{a}\left(t_{R}\right)+\left.\frac{\mathrm{d} \boldsymbol{v}_{a}}{\mathrm{~d} t}\right|_{t_{R}} \Delta t_{a}\right], \quad d e n_{b}=1-\frac{1}{c} \hat{R}_{b} \cdot\left[\boldsymbol{v}_{b}\left(t_{R}\right)+\left.\frac{\mathrm{d} \boldsymbol{v}_{b}}{\mathrm{~d} t}\right|_{t_{R}} \Delta t_{b}\right] \\
\Delta t_{a}=\frac{1}{c}\left[\hat{R} \cdot \boldsymbol{r}_{a}\right], \Delta t_{b}=\frac{1}{c}\left[\hat{R} \cdot \boldsymbol{r}_{b}\right]
\end{gathered}
$$

Equation (16a) is rearranged as $\boldsymbol{A}(\boldsymbol{R}, t)=\boldsymbol{p}+\boldsymbol{F}$ : the term containing the linear momentum plus the one containing the inertial force.

$$
\begin{array}{r}
\boldsymbol{p}_{\text {Tran }}=\frac{G \omega r}{c^{2} R} \eta M\left(\frac{1}{d e n_{a}}-\frac{1}{d e n_{b}}\right)\left(-\cos \theta \sin \left(\omega \cdot t_{R}\right) \hat{\theta}+\cos \left(\omega \cdot t_{R}\right) \hat{\phi}\right) \\
=c_{p} p_{1}(t) \boldsymbol{p}_{2}(t) \\
\boldsymbol{F}_{\text {Tran }}=-\frac{G \omega^{2} r^{2}}{2 c^{3} R} \eta\left(\frac{m_{b}}{d e n_{a}}+\frac{m_{a}}{d e n_{b}}\right) \sin \theta\left[\cos \theta\left(1+\cos \left(2 \omega \cdot t_{R}\right)\right) \hat{\theta}+\sin \left(2 \omega \cdot t_{R}\right) \hat{\phi}\right] \\
=-c_{F} F_{1}(t) \boldsymbol{F}_{2}(t)
\end{array}
$$

where,

$$
\begin{gathered}
\operatorname{den}_{a}=1+\frac{\omega \cdot r_{a}}{c} \sin \theta \sin \left(\omega \cdot t_{R}\right)+\frac{\omega^{2} r_{a}^{2}}{2 c^{2}} \sin ^{2} \theta\left(1+\cos \left(2 \omega \cdot t_{R}\right)\right) \\
\operatorname{den}_{b}=1-\frac{\omega \cdot r_{b}}{c} \sin \theta \sin \left(\omega \cdot t_{R}\right)+\frac{\omega^{2} r_{b}^{2}}{2 c^{2}} \sin ^{2} \theta\left(1+\cos \left(2 \omega \cdot t_{R}\right)\right)
\end{gathered}
$$

Equations (12)-(15) were used to obtain Equation (17)-(18). The Cartesian unit vectors were expressed in terms of the spherical ones with the choice of $\phi=0$ as in [1]. To obtain Equation (19) it was assumed that $\hat{R}_{a} \approx \hat{R}_{b} \approx \hat{R}$.

Note that even for $r_{a}=r_{b}, d e n_{a} \neq d e n_{b}$, which is consistent with having contribution from $\boldsymbol{p}_{\text {Tran }}$ for equal masses.

$\hat{\theta}, \hat{\phi}$ : Are the unit vectors (spherical coordinates) perpendicular to the direction of the observation vector $\boldsymbol{R} . \eta=\frac{m_{a} m_{b}}{\left(m_{a}+m_{b}\right)^{2}}$

Note that if $d e n_{a}=d e n_{b}=1$, Equation (47) of [1] is recovered except for the negative sign in Equation (18).

Note also that for small $\mathrm{v}$ even though $\frac{1}{d e n_{a}}-\frac{1}{d e n_{b}}$ is small and $\frac{m_{b}}{d e n_{a}}+\frac{m_{a}}{d e n_{b}} \approx M, \frac{\boldsymbol{p}_{\text {Tran }}}{\boldsymbol{F}_{\text {Tran }}}$ is, unexpectedly, not necessarily negligible.

The radiation field is therefore:

$$
\boldsymbol{g}_{\text {rad }}=-\frac{\partial \boldsymbol{A}_{\text {Tran }}}{\partial t}=-c_{p}\left(\frac{\partial p_{1}}{\partial t} \boldsymbol{p}_{2}+p_{1} \frac{\partial \boldsymbol{p}_{2}}{\partial t}\right)+c_{F}\left(\frac{\partial F_{1}}{\partial t} \boldsymbol{F}_{2}+F_{1} \frac{\partial \boldsymbol{F}_{2}}{\partial t}\right)
$$




$$
\begin{gathered}
\frac{\partial p_{1}}{\partial t} \boldsymbol{p}_{2}=\omega\left(\frac{n_{a}}{d e n_{a}^{2}}+\frac{n_{b}}{d e n_{b}^{2}}\right)\left(-\cos \theta \sin \left(\omega \cdot t_{R}\right) \hat{\theta}+\cos \left(\omega \cdot t_{R}\right) \hat{\phi}\right) \\
n_{a}=-\frac{\omega \cdot r_{a}}{c} \sin \theta \cos \left(\omega \cdot t_{R}\right)+\frac{\omega^{2} r_{a}^{2}}{c^{2}} \sin ^{2} \theta \sin \left(2 \omega \cdot t_{R}\right) \\
n_{b}=-\frac{\omega \cdot r_{b}}{c} \sin \theta \cos \left(\omega \cdot t_{R}\right)-\frac{\omega^{2} r_{b}^{2}}{c^{2}} \sin ^{2} \theta \sin \left(2 \omega \cdot t_{R}\right) \\
p_{1} \frac{\partial \boldsymbol{p}_{2}}{\partial t}=\omega\left(\frac{1}{d e n_{a}}-\frac{1}{d e n_{b}}\right)\left(-\cos \theta \cos \left(\omega \cdot t_{R}\right) \hat{\theta}-\sin \left(\omega \cdot t_{R}\right) \hat{\phi}\right) \\
\frac{\partial F_{1}}{\partial t} \boldsymbol{F}_{2}=\omega\left(\frac{m_{b} n_{a}}{d e n_{a}^{2}}-\frac{m_{a} n_{b}}{d e n_{b}^{2}}\right) \sin \theta\left[\cos \theta\left(1+\cos \left(2 \omega \cdot t_{R}\right)\right) \hat{\theta}+\sin \left(2 \omega \cdot t_{R}\right) \hat{\phi}\right] \\
F_{1} \frac{\partial \boldsymbol{F}_{2}}{\partial t}=2 \omega\left(\frac{m_{b}}{d e n_{a}}+\frac{m_{a}}{d e n_{b}}\right) \sin \theta\left[-\cos \theta \sin \left(2 \omega \cdot t_{R}\right) \hat{\theta}+\cos \left(2 \omega \cdot t_{R}\right) \hat{\phi}\right]
\end{gathered}
$$

Note that $\omega, r_{a}, r_{b}, r$, were considered constants for the calculation of the derivatives in Equation (20). If the terms containing the derivatives in Equation (20) are divided by $\omega$, the quasi-constants are redefined as $c_{p}=\frac{G \omega^{2} r}{c^{2} R} \eta M$, $c_{F}=\frac{G \omega^{3} r^{2}}{2 c^{3} R} \eta$ which helps to see that making $c_{p}=0$ and $F_{1}(t)=M$, Equation (48) of [1] (with opposite sign) is obtained.

The radiated power per unit area is

$$
\begin{gathered}
\boldsymbol{S}=\frac{c}{4 \pi G} \boldsymbol{g}_{\text {rad }}^{2} \hat{\boldsymbol{S}}=\frac{c}{4 \pi G}\left(\boldsymbol{g}_{\text {rad_t }_{-}}^{2}+\boldsymbol{g}_{\text {rad }_{-} \hat{\phi}}^{2}\right) \hat{\boldsymbol{S}}, \text { the total power radiated is } \\
\frac{\mathrm{d} E}{\mathrm{~d} t}=\int \boldsymbol{S} \cdot \mathrm{d} \boldsymbol{A}, \mathrm{d} \boldsymbol{A}=R^{2} \sin \theta \mathrm{d} \theta \mathrm{d} \phi \hat{S} \\
\frac{\mathrm{d} E}{\mathrm{~d} t}=\left.\frac{\mathrm{d} E}{\mathrm{~d} t}\right|_{\hat{\theta}}+\left.\frac{\mathrm{d} E}{\mathrm{~d} t}\right|_{\hat{\phi}},\left.\frac{\mathrm{d} E}{\mathrm{~d} t}\right|_{\hat{\theta}}=\frac{c R^{2}}{2 G} \int_{0}^{\pi} \boldsymbol{g}_{\text {rad }_{-} \hat{\theta}}^{2} \sin \theta \mathrm{d} \theta,\left.\frac{\mathrm{d} E}{\mathrm{~d} t}\right|_{\hat{\phi}}=\frac{c R^{2}}{2 G} \int_{0}^{\pi} \boldsymbol{g}_{\text {rad } \hat{\phi}}^{2} \sin \theta \mathrm{d} \theta
\end{gathered}
$$

The integrals will be determined numerically.

\subsection{Gravitational Orbit Decay}

The total classical mechanical energy of the binary system is [1]

$$
U_{\text {Tot }}=\frac{1}{2} m_{a} v_{a}^{2}+\frac{1}{2} m_{b} v_{b}^{2}-G \frac{m_{a} m_{b}}{r}=-G \frac{m_{a} m_{b}}{2 r} \text {. Kepler's } 3^{\text {rd }} \text { law for circular or- }
$$

bits was used to obtain the right hand side equality. Note that the classical EM potential energy is also written with an equation similar to the $3^{\text {rd }}$ term of left hand side [7].

From energy conservation follows $\frac{\mathrm{d} U_{\text {tot }}}{\mathrm{d} t}=-\frac{\mathrm{d} E}{\mathrm{~d} t}$ so the orbit decay is determined from

$$
\dot{r}=-\frac{2 r^{2}}{G m_{a} m_{b}} \frac{\mathrm{d} E}{\mathrm{~d} t}
$$

To consider the extended $3 \mathrm{KL}$, the mechanical energy equation is written as 


$$
\begin{aligned}
U_{\text {Tot }}=\frac{1}{2} \eta M \omega^{2} r^{2}-G \frac{m_{a} m_{b}}{r} & \text {, therefore } \\
& \frac{\mathrm{d} U_{t o t}}{\mathrm{~d} t}=\eta M\left(\omega \dot{\omega} r^{2}+\omega^{2} r \dot{r}\right)+G \frac{m_{a} m_{b}}{r^{2}} \dot{r}
\end{aligned}
$$

From Equation (8)

$$
\begin{aligned}
\omega^{2} & =\frac{1}{\frac{\left(\eta_{b} \eta_{a}+\eta_{a}^{2}\right) r^{2}}{c^{2}}+\frac{r^{3}}{G M}}+\frac{\frac{\eta_{b}\left(\eta_{a}^{3}-\eta_{a}^{2}\right) r^{4}}{c^{4}}}{\frac{\left(\eta_{b} \eta_{a}+\eta_{a}^{2}\right) r^{2}}{c^{2}}+\frac{r^{3}}{G M}} \omega^{4} \\
& =\frac{1}{d}+\frac{n}{d} \omega^{4}=f_{0}(r)+f_{4}(r) \omega^{4}
\end{aligned}
$$

Taking the derivative in both sides:

$$
\begin{gathered}
2 \omega \dot{\omega}=\dot{f}_{0}(r)+\dot{f}_{4}(r) \omega^{4}+f_{4}(r) 4 \omega^{3} \dot{\omega} \Rightarrow \\
\left(2 \omega-4 f_{4}(r) \omega^{3}\right) \dot{\omega}=\dot{f}_{0}(r)+\dot{f}_{4}(r) \omega^{4} \Rightarrow \\
\dot{\omega}=\frac{\dot{f}_{0}(r)+\dot{f}_{4}(r) \omega^{4}}{2 \omega-4 f_{4}(r) \omega^{3}} .
\end{gathered}
$$

where,

$$
\begin{gathered}
\dot{f}_{0}(r)=-\frac{\dot{d}}{d^{2}}=-\frac{1}{d^{2}}\left(2 \frac{\left(\eta_{b} \eta_{a}+\eta_{a}^{2}\right) r}{c^{2}}+3 \frac{r^{2}}{G M}\right) \dot{r}=q_{0} \dot{r} \\
\dot{f}_{4}(r)=\frac{\dot{n} d-n \dot{d}}{d^{2}}=\frac{4 \frac{\eta_{b}\left(\eta_{a}^{3}-\eta_{a}^{2}\right) r^{3}}{c^{4}} d \dot{r}-n\left(2 \frac{\left(\eta_{b} \eta_{a}+\eta_{a}^{2}\right) r}{c^{2}} \dot{r}+3 \frac{r^{2}}{G M} \dot{r}\right)}{d^{2}}=q_{4} \dot{r} .
\end{gathered}
$$

Therefore

$$
\begin{aligned}
& \dot{\omega}=\frac{q_{0}(r)+q_{4}(r) \omega^{4}}{2 \omega-4 f_{4}(r) \omega^{3}} \dot{r} \text { substituting into Equation (22) leads to: } \\
& \frac{\mathrm{d} U_{t o t}}{\mathrm{~d} t}=\left(\eta M\left(\omega \frac{q_{0}(r)+q_{4}(r) \omega^{4}}{2 \omega-4 f_{4}(r) \omega^{3}} r^{2}+\omega^{2} r\right)+G \frac{m_{a} m_{b}}{r^{2}}\right) \dot{r}=q \dot{r}=-\frac{\mathrm{d} E}{\mathrm{~d} t}
\end{aligned}
$$

\subsection{Gravitational Wave Signal}

The acceleration of the beam splitter ( $\mathrm{x}$-arm) for the [1] model of the LIGO-VIRGO detectors is written as

$$
\ddot{x}_{1}=\boldsymbol{g}_{\text {rad }}\left(t_{1}\right) \cdot \hat{x}=\boldsymbol{g}_{\text {rad_ } \hat{\theta}}\left(t_{1}\right) \cdot \hat{x}=\boldsymbol{g}_{\text {rad_êt }}\left(t_{1}\right) \sin \beta
$$

and for the mirror:

$$
\ddot{x}_{2}=\boldsymbol{g}_{\text {rad }}\left(t_{2}\right) \cdot \hat{x}=\boldsymbol{g}_{\text {rad_t }}\left(t_{2}\right) \cdot \hat{x}=\boldsymbol{g}_{\text {rad_t }}\left(t_{2}\right) \sin \beta
$$

where

$\beta:$ is the angle between the wave propagation direction and the interferometer $\mathrm{x}$ arm.

$t_{1}, t_{2}:$ are the arrival time of the wave at the beam splitter and mirror respectively. 


$$
t_{2}=t_{1}+L \cos \beta / c
$$

$L:$ is the interferometer arm.

Equations (24a)-(b) are wished to be solved numerically. Assuming the existence of an undistorted convergent solution, the relative displacement of the two masses in the $\mathrm{x}$ arm is determined as $\Delta L_{x}=x_{2}-x_{1}$. It can be shown that the relative displacement for the $\mathrm{Y}$ arm is $\Delta L_{y}=y_{2}-y_{1}=-\Delta L_{x}$. The interferometer strain signal is then expressed as $h=\frac{\Delta L_{x}-\Delta L_{y}}{L}$. For simplicity the two arm lengths were assumed to be the same in the model of [1].

\section{Impact of the ENET Relativistic Acceleration on the Gravitational Orbit Decay}

\subsection{Extension of Kepler's $3^{\text {rd }}$ Law Using the ENET Acceleration in the Gravitational Fields}

When the speed of components of the binary system is a significant percent of the speed of light a relativistic theory should be used to address the problems that cannot be explained with Newtonian theory, for example, the intrinsic (two body problem) perihelion precession of the planets (specially Mercury).

It is well-known that Einstein's special theory of relativity (STR) does not yield precession values consistent with experiments, that is why (probably) Einstein, based on his concept of curved space-time (unlike the flat space-time of STR), developed the remarkable GTR which does yield results in agreement with experiments and predicted other currently verified phenomena.

Ref [4] however derived a relativistic inertial acceleration (ENET), which is based on the application of two consecutive boosts of the apparent time dilation and length contraction (concepts which are supported by the Michelson-Morley experiment, the Lorentz-Fitzgerald transformation, and STR) to the Newtonian concept of time and space interval. The so obtained relativistic acceleration also yields results consistent with the experimental values of the precession of planets.

The ENET acceleration is written as $\boldsymbol{a}=\left(1-\beta^{2}\right)^{3} \boldsymbol{a}_{N}$ where $\boldsymbol{a}_{N}$ is the Newtonian acceleration and $\beta=v / c$.

Using this acceleration in Equations (2)-(3), Equations (4)-(5) are reproduced. Equation (6) becomes $f=\omega^{3}\left(r_{b}^{3}-r r_{b}^{2} g_{b}\right)$ and Equation (8) becomes

$$
\begin{gathered}
-\frac{\eta_{b}\left(\eta_{a}^{3}-\eta_{a}^{2} g_{b}\right) r^{4}}{c^{4}} \omega^{4}+\left(\frac{\left(\eta_{b} \eta_{a}+\eta_{a}^{2}\right) r^{2}}{c^{2}}+\frac{r^{3}}{G M} g_{a}\right) \omega^{2}-1=0 \\
g_{b}=\left(1-\frac{\left(\omega \cdot \eta_{a} \cdot r\right)^{2}}{c^{2}}\right)^{3}, g_{a}=\left(1-\frac{\left(\omega \cdot \eta_{b} \cdot r\right)^{2}}{c^{2}}\right)^{3}
\end{gathered}
$$

Equation (25) is to be solved numerically. Note that making $c=\infty \mathrm{K} 3 \mathrm{~L}$ for circular orbits is here also recovered.

From Equation (25): 


$$
\begin{aligned}
\omega^{2} & =\frac{1}{\frac{\left(\eta_{b} \eta_{a}+\eta_{a}^{2}\right) r^{2}}{c^{2}}+\frac{r^{3}}{G M} g_{a}}+\frac{\frac{\eta_{b}\left(\eta_{a}^{3}-\eta_{a}^{2} g_{b}\right) r^{4}}{c^{4}}}{\frac{\left(\eta_{b} \eta_{a}+\eta_{a}^{2}\right) r^{2}}{c^{2}}+\frac{r^{3}}{G M} g_{a}} \omega^{4} \\
& =\frac{1}{d}+\frac{n}{d} \omega^{4}=f_{0}(r)+f_{4}(r) \omega^{4}
\end{aligned}
$$

From which as before $\dot{\omega}=\frac{\dot{f}_{0}(r)+\dot{f}_{4}(r) \omega^{4}}{2 \omega-4 f_{4}(r) \omega^{3}}$ where,

$$
\begin{gathered}
\dot{f}_{0}(r)=-\frac{\dot{d}}{d^{2}}, \quad \dot{d}=2 \frac{\left(\eta_{b} \eta_{a}+\eta_{a}^{2}\right) r}{c^{2}} \dot{r}+3 \frac{r^{2}}{G M} \dot{r} g_{a}+\frac{r^{3}}{G M} \dot{g}_{a} \\
\dot{g}_{a}=-6 \frac{\eta_{b}^{2}}{c^{2}}\left(1-\frac{\left(\omega \eta_{b} r\right)^{2}}{c^{2}}\right)^{2} \omega r(\dot{\omega} r+\omega \dot{r})=k_{a}(\omega \dot{r}+r \dot{\omega})
\end{gathered}
$$

So $\dot{f}_{0}(r)=q_{0} \dot{r}+q_{g a} \dot{\omega}$

$q_{0}:$ is the resultant factor from collecting the multipliers of $\dot{r}$ $q_{g a}:$ is the resultant factor from collecting the multipliers of $\dot{\omega}$

$$
\dot{f}_{4}(r)=\frac{\dot{n} d-n \dot{d}}{d^{2}}, \quad \dot{n}=\frac{\eta_{b}}{c^{4}}\left(-\eta_{a}^{2} \dot{g}_{b} r^{4}+4\left(\eta_{a}^{3}-\eta_{a}^{2} g_{b}\right) r^{3} \dot{r}\right), \quad \dot{g}_{b}=k_{b}(\omega \dot{r}+r \dot{\omega})
$$

So $\dot{f}_{4}(r)=q_{4} \dot{r}+q_{g b} \dot{\omega}$

Therefore

$$
\begin{gathered}
\dot{\omega}=\frac{q_{0} \dot{r}+q_{g a} \dot{\omega}+\left(q_{4} \dot{r}+q_{g b} \dot{\omega}\right) \omega^{4}}{2 \omega-4 f_{4}(r) \omega^{3}} \Rightarrow \\
\left(2 \omega-4 f_{4}(r) \omega^{3}-q_{g a}-q_{g b} \omega^{4}\right) \dot{\omega}=\left(q_{0}+q_{4} \omega^{4}\right) \dot{r} \\
\Rightarrow \dot{\omega}=\frac{q_{0}+q_{4} \omega^{4}}{2 \omega-4 f_{4}(r) \omega^{3}-q_{g a}-q_{g b} \omega^{4}} \dot{r} \quad \text { Substituting into Equation (22) leads }
\end{gathered}
$$

to:

$$
\begin{aligned}
\frac{\mathrm{d} U_{t o t}}{\mathrm{~d} t} & =\eta M\left(\omega \frac{q_{0}+q_{4} \omega^{4}}{2 \omega-4 f_{4}(r) \omega^{3}-q_{g a}-q_{g b} \omega^{4}} r^{2} \dot{r}+\omega^{2} r \dot{r}\right)+G \frac{m_{a} m_{b}}{r^{2}} \dot{r} \\
& =q \dot{r}=-\frac{\mathrm{d} E}{\mathrm{~d} t}
\end{aligned}
$$

\section{Computational Results and Analysis}

Figure 1 shows the angular speed as function of radius (in units of $r_{s}=\frac{2 G M}{c^{2}}$, the Schwarzschild radius, for a system of equal masses with total mass of $70 M_{\text {sun }}$. The mass separation values were just generated. NK1 is the Kepler's $3^{\text {rd }}$ law. NK2 is the extended K3L considering the E\&M analogy (Equation (8)). NK3 is the extended $\mathrm{K} 3 \mathrm{~L}$ considering the $\mathrm{E} \& \mathrm{M}$ analogy and replacing the Newtonian acceleration with the relativistic acceleration of ENET (Equation (25) was solved iteratively by isolating the quadratic term). Note that only for mass separations significantly smaller than $r_{s}$ they drastically differ among themself. It is curious 
that NK2 curvature is smaller than the NK1's one.

Figures 2-5 show results also for a system of equal masses with total mass of $70 M_{\text {sun }}$ The total radiated power, $\frac{\mathrm{d} E}{\mathrm{~d} t}$, was calculated numerically using 100 angular partitions.

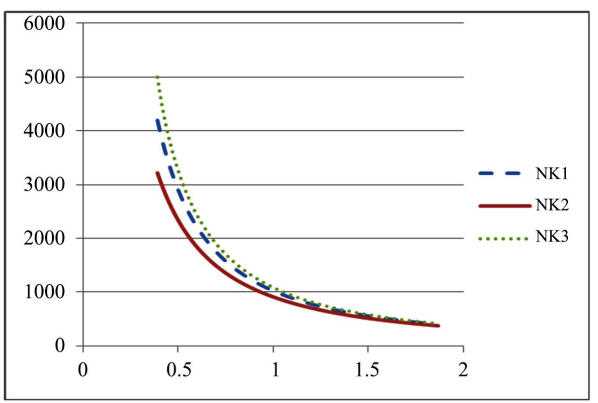

Figure 1. Angular speed (1/s) vs. mass separation (rs).

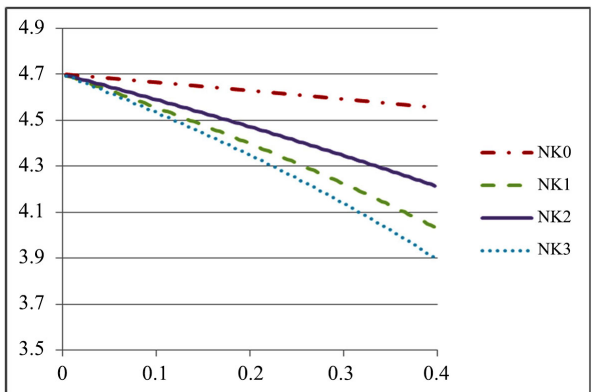

Figure 2. Mass separation (rs) vs. time (s).

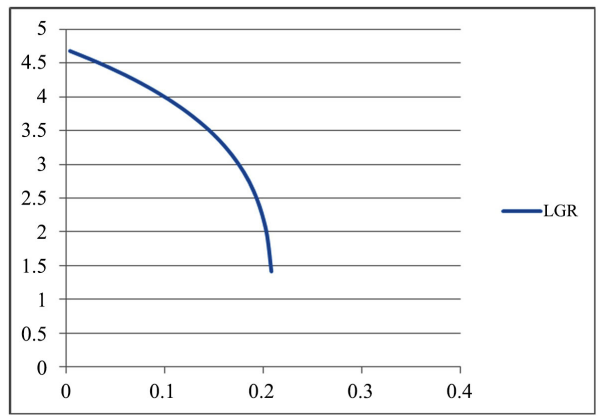

Figure 3. Mass separation (rs) vs. time (s).

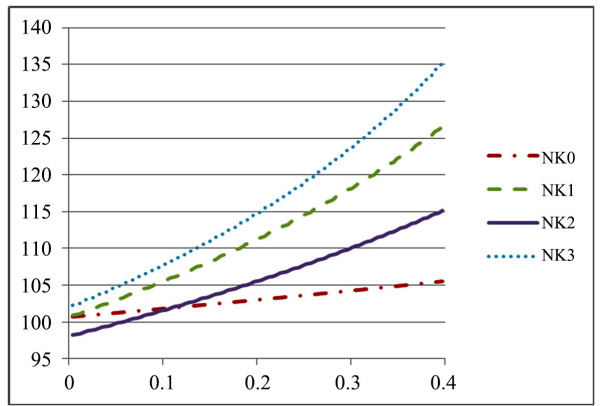

Figure 4. Angular speed (1/s) vs. time (s). 


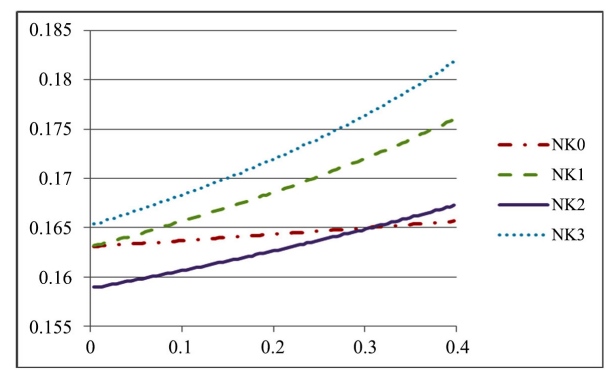

Figure 5. Speed (c) vs. time (s).

Figure 2 shows the mass separation as a function of time (orbit decay). NK0 is the model of [1] [2] where the mass separation is calculated from

$$
r^{4}=r_{0}^{4}-N \eta c r_{s}^{3}\left(t-t_{0}\right), \quad N=2 / 5
$$

Equation (27) does not consider the correction related with the ratio of the binary speed to the speed of light in vacuum. From that Figure can be seen that the extended models yield faster orbit decay than NK0 results. NK1 uses K3L in the extended gravitational model and the results were obtained by solving Equation (21). NK2 model is represented by Equation (23) and NK3 results are determined from Equation (26). Numerical derivatives (finite difference) were used to solve Equation (21), (23), and (26) with an integration step of $1.0 \times 10^{-5}$ seconds.

Figure 3 shows the results of the LGR (using the same equation used in NK0 model but with $N=32 / 5$. From that Figure can be seen a very fast orbit decay in just $0.2 \mathrm{sec}$.(compare it with Figure 2) For time slightly larger, the separation becomes negative probably an indication that the ring down and merger of the binaries should be considered. Figure 4 and Figure 5 show the angular speed $(1 / s)$ and the speed of the binaries respectively

Figure 6 shows the radiation fields yielded by NK0:

$$
\boldsymbol{g}_{\text {rad_ } \__{-}}=\frac{G \eta M \omega^{3} r^{2}}{2 c^{3} R} \sin 2 \theta \sin \left(2 \omega \cdot t_{R}\right)
$$

and NK1 (Equation (20): the same for NK2 and NK3) for a system of equal masses with total mass of $65 M_{\text {sun }}$ and an initial mass separation of $5.3 r_{s}$. These data were inferred from the GW150914 black hole merging event [2] (except for the equality of the masses assume here). The following data were used also: $R=410 \mathrm{Mpc}, \theta=150^{\circ}, L=4000 \mathrm{~m}, \beta=45^{\circ}$ [1] [8].

Note that not only the amplitude of NK1 is significantly larger than the NK0 but the number of positive peaks is also greater ( 31 vs. 23$)$ which is an indication that the frequency increase is also larger. The results for NK2 and NK3 are as expected (NK2 amplitude smaller than NK1 and NK3 larger than NK1).

A rigorous calculation of the wave form was not made because a convergent and correct numerical solution for the displacement has not yet been found (not even for NK0 which has an analytic simple solution). The reason for that has not yet been determined. 


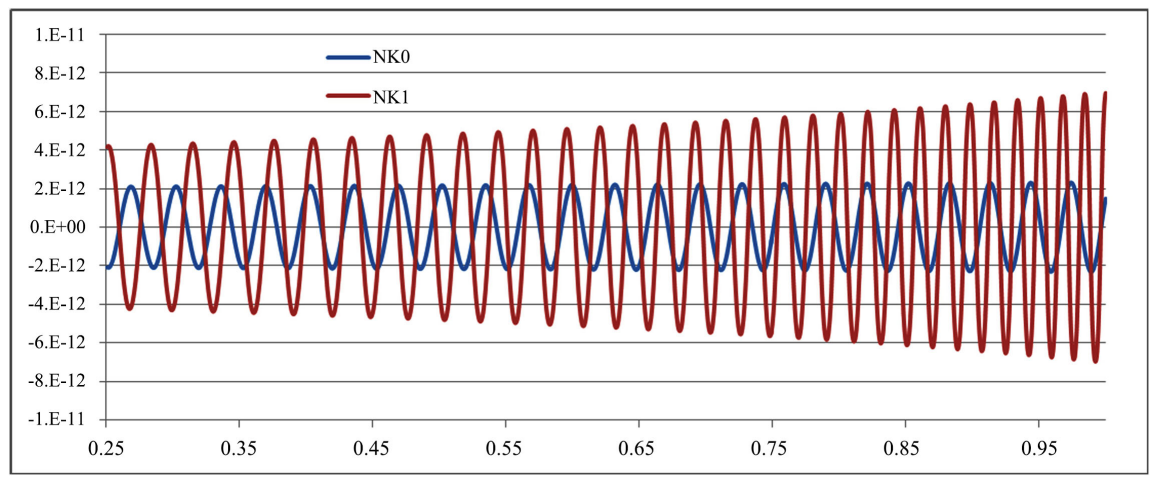

Figure 6. Radiation field $\left(\mathrm{m} / \mathrm{s}^{2}\right)$ vs. time (s). NK1 is the one with the largest amplitude.

It could be worthy to check if the radiation field could be approximated as a generalization of the Equation (28) as

$\boldsymbol{g}_{\text {rad_ } \hat{\theta}}=n_{a w} \frac{G \eta M \omega^{3} r^{2}}{2 c^{3} R} \sin 2 \theta \sin \left(n_{k w} 2 \omega \cdot t_{R}\right)$ to determine $n_{k a} \quad n_{k w}$. If the approximation is reasonable then the solution is obtained as $x=-\frac{\boldsymbol{g}_{\text {rad } \_\hat{\theta}} \sin (\beta)}{\left(n_{k w} 2 \omega\right)^{2}}$. Note that the objective is to obtain, if possible, an $n_{k w}$ that makes the approximated radiation field to be reasonably in phase with the original one.

Figure 7 shows the results of the wave form (strain signal) calculation for NK0 and for a gross approximation of NK1 using $n_{k w}=1$ to have a tentative idea of the amplitude of the strain signal $(h)$. The results for NK2 and NK3 are as expected. Note that the GR model predicts the beginning of the merger about 0.4 sec. into the transient [1]. The calculation in Figure 7 was extended to 1 sec. just to see the trends better.

It is notified that using the accumulated phase equation,

$\Phi\left(t_{R}\right)=\int_{t_{0}}^{t} \omega\left(t^{\prime}\right) \mathrm{d} t^{\prime} \approx \sum_{1}^{N t} \omega_{i} \Delta t_{i}=\Delta t \sum_{1}^{N t} \omega_{i}$, in place of $\omega t_{R}$ in any trigonometric function in Equation (20), results in shapes and amplitudes similar to Figure 6 and Figure 7, however a shift in phase is observed.

For curiosity it was checked if values of $N$ in Equation (27) can be obtained that reproduce the results of NK1, NK2, and NK3. The obtained (by trial and error targeting the final value of $\mathrm{r}$ ) values are $7.55 / 5,6 / 5$ and $8.56 / 5$ respectively. Those values reproduced the whole profile of the models accurately (at least up to the first digit after the decimal point). Using those values of $N$ in the previous example good profiles are also obtained. These results encourage the search for $n_{k w}$ and, if needed, the search for simpler equations for the mass separation, irradiated power and the radiation field.

The last application considered was the calculation of the period decay of the Hulse-Taylor binary pulsar (PSR $1913+16$ ) assuming circular orbits. The data for this simulation are [9]: pulsar mass: $1.4408 M_{\text {sun }}$, companion mass: 1.3873 $M_{\text {sunn }}$, Orbital period: 0.323 days. The period decay was calculated numerical as 


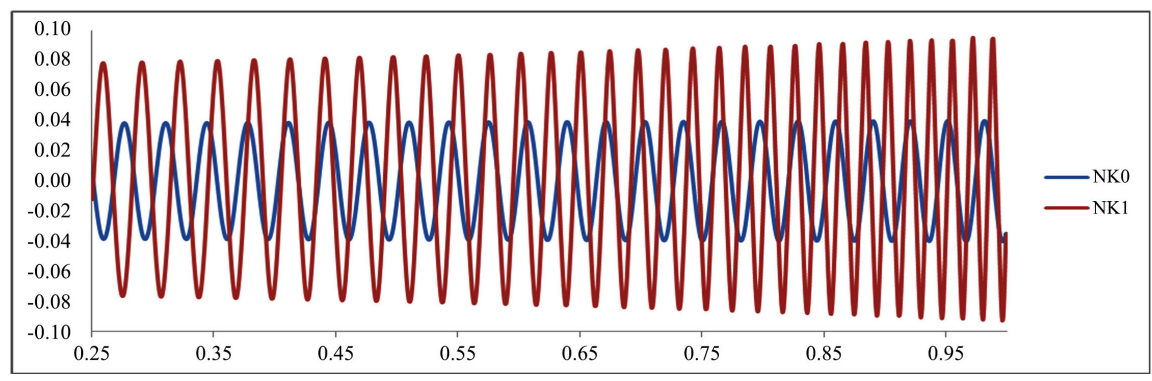

Figure 7. Strain signal (multiplied by 1021) vs. time (s). NK1 is the one with the largest amplitude.

$\left.\frac{\mathrm{d} T}{\mathrm{~d} t}\right|_{i}=\frac{T_{i}-T_{i-1}}{\Delta t}, T_{i}=2 \pi / \omega_{i}$. The time step used was 0.1 day. Even though the concept of derivative requires small time step, the change rate of the period is so small that if the time step is too small the compiler will produce $\frac{\mathrm{d} T}{\mathrm{~d} t}=0$. The calculation span was 365 days. The results of the average values are: $-1.26 \mathrm{E}-14$, $-5.02 \mathrm{E}-14,-6.72 \mathrm{E}-14$, and $-5.02 \mathrm{E}-14$ for NK0 $(N=2 / 5)$, NK1, NK2, and NK3 respectively. The LGR $(N=32 / 5)$ result was $-2.02 \mathrm{E}-13$ which coincides with the theoretical value calculated from [9] [10]

$$
\frac{\mathrm{d} T}{\mathrm{~d} t}=\frac{192 \pi m_{a} m_{b}}{5 c^{5}\left(m_{a}+m_{b}\right)^{1 / 3}}\left(\frac{2 \pi G}{T}\right)^{5 / 3}
$$

\section{Summary and Concluding Remarks}

The Kepler $3^{\text {rd }}$ law was extended considering the gravitational field (in analogy to $\mathrm{E} \& \mathrm{M})$ produced from the circular motion of the masses of a binary system. The Newtonian acceleration in the field equation was replaced with the ENET acceleration. It was found out that the increase of the angular speed with the decrease of the mass separation is less drastic for the electromagnetic analogy than for the K3L case. The use of the ENET acceleration however yielded a more drastic profile than the $\mathrm{K} 3 \mathrm{~L}$ results.

A previous gravito-electromagnetic model developed for non-relativistic speed was extended to cover relativistic speeds. It was found out that even for low speeds the linear-momentum term was not negligible. The extended model yielded faster orbital decay than the non-extended one. The use of the extended K3L using ENET yielded even faster orbit decay but slower than the results of LGR. Similar results were obtained when the gravitational radiation fields were compared.

The period decay (for circular orbit) of the Hulse and Taylor pulsar binary was simulated; the extended models yielded larger period decay than the non-extended one but smaller than the results of LGR for circular orbit. It could be worthy to use elliptical orbits to see the impact on this comparison. It is notified that calculations (not described here) of the perihelion shift of the Hulse and Taylor binary pulsar using Newtonian gravitation with the ENET acceleration 
yielded results in agreement with experiment and GR.

\section{Acknowledgements}

I thank Dr. Robert C. Hilborn for his feedbacks during my learning of his tutorial and during this paper-development, for reading a draft of this paper and for suggesting the accumulated phase calculations. I thank also the anonymous reviewer for suggestions to improve the presentation of this paper.

\section{Conflicts of Interest}

The author declares no conflicts of interest regarding the publication of this paper.

\section{References}

[1] Hilborn, R.C. (2017) Gravitational Waves from Orbiting Binaries without General Relativity: A Tutorial.

[2] Hilborn, R.C. (2018) Gravitational Waves from Orbiting Binaries without General Relativity. American Journal of Physics, 86, 186. https://doi.org/10.1119/1.5020984

[3] Svidzinsky, A.A. and Hilborn, R.C. (2018) GW170817 Event Rules out General Relativity in Favor of Vector Gravity.

[4] Quintero-Leyva, B. (2016) An Extended Newtonian Theory for Gravitational Bound Systems. Open Access Library Journal, 3, e2678.

https://doi.org/10.4236/oalib.1102678

[5] Griffiths, D.J. (1999) Introduction to Electrodynamics. 4th Edition, Prentice-Hall, Englewood Cliffs.

[6] https://physicspages.com/Griffiths\%20EM.html

[7] Gutierrez, P. (2003) Physics 5153. Classical Mechanics Velocity Dependent Potentials. An Internet Resource.

[8] Abbott, B.P., et al. (2016) Properties of Binary Black Hole Merger GW150914. Physical Review Letters, 116, Article ID: 241102. https://doi.org/10.1103/PhysRevLett.116.241102

[9] Ninive, P.H. (2009) Chameleon Fields and Gravitational Waves. Master of Science Thesis, Institute of Theoretical Astrophysics, University of Oslo, Oslo.

[10] Taylor, J.H. and Weisberg, J.M. (1982) A New Test of General Relativity: Gravitational Radiation and the Binary Pulsar PSR 1913+16. The Astronomical Journal, 253, 908-920. https://doi.org/10.1086/159690 